\title{
HERMITE-HADAMARD TYPE INEQUALITIES FOR HARMONICALLY CONVEX FUNCTIONS VIA KATUGAMPOLA FRACTIONAL INTEGRALS
}

\author{
İLKER MUMCU, ERHAN SET, AND AHMET OCAK AKDEMIR \\ Received 25 October, 2018
}

\begin{abstract}
In this work, firstly, we established Hermite-Hadamard's inequalities for harmonically convex functions via Katugampola fractional integrals. Then we give some Hermite-Hadamard type inequalities of these classes functions.
\end{abstract}

2010 Mathematics Subject Classification: 26A33; 26A51; 26D10

Keywords: Hermite-Hadamard inequality, Riemann-Liouville fractional integrals, Katugampola fractional integrals

\section{INTRODUCTION AND PRELIMINARIES}

We will start with a definition of mathematical analysis that has a high degree precedence for the inequality theory.

A function $f: I \subseteq \mathbb{R} \rightarrow \mathbb{R}$ is said to be convex if the inequality

$$
f(\lambda u+(1-\lambda) v) \leq \lambda f(u)+(1-\lambda) f(v)
$$

holds for all $u, v \in I$ and $\lambda \in[0,1]$.

This definition has been used in the celebrated Hermite-Hadamard inequality;

Let $f: I \subseteq \mathbb{R} \rightarrow \mathbb{R}$ be a convex function and $a, b \in I$ with $a<b$, then

$$
f\left(\frac{a+b}{2}\right) \leq \frac{1}{b-a} \int_{a}^{b} f(x) d x \leq \frac{f(a)+f(b)}{2} .
$$

In addition to giving upper and lower bounds for the mean value of a convex function, this double inequality has many applications.

Convexity plays an important role in different fields of pure and applied sciences. In recent years we have noticed that theory of convexity developed rapidly. Consequently several new generalizations of convex functions have been proposed in the literature. Recently Işcan [4] introduced the notion of harmonic convex function. 
Definition 1. Let $I \subset \mathbb{R} /\{0\}$ be a real interval. A function $f: I \rightarrow \mathbb{R}$ is said to be harmonically convex, if

$$
f\left(\frac{x y}{t x+(1-t) y}\right) \leq t f(y)+(1-t) f(x)
$$

for all $x, y \in I$ and $t \in[0,1]$.

The following theorem involve a different variant of Hadamard's inequality for harmonically convex functions.

Theorem 1 ([4]). Let $I \subset \mathbb{R} /\{0\} \rightarrow \mathbb{R}$ be a harmonically convex function and $a, b \in I$ with $a<b$. If $f \in L[a, b]$ then the following inequalities hold.

$$
f\left(\frac{2 a b}{a+b}\right) \leq \frac{a b}{b-a} \int_{a}^{b} \frac{f(x)}{x^{2}} d x \leq \frac{f(a)+f(b)}{2} .
$$

To prove our results, we will use the following concepts and definitions. The Beta function [11, p.18]:

$$
B(a, b)=\frac{\Gamma(a) \Gamma(b)}{\Gamma(a+b)}=\int_{0}^{1} t^{a-1}(1-t)^{b-1} d t, \quad a, b>0,
$$

where $\Gamma(\alpha)=\int_{0}^{\infty} e^{-t} u^{\alpha-1} d u$ is Gamma function.

The hypergeometric function [7]:

$$
{ }_{2} F_{1}(a, b ; c ; z)=\frac{1}{\beta(b, c-b)} \int_{0}^{1} t^{b-1}(1-t)^{c-b-1}(1-z t)^{-a} d t, c>b>0, z<1 .
$$

Lemma 1 ([10]). For $0<\alpha \leq 1$ and $0 \leq a<b$, we have

$$
\left|a^{\alpha}-b^{\alpha}\right| \leq(b-a)^{\alpha} \text {. }
$$

Definition 2. Let $f \in L_{1}[a, b]$. The Riemann-Liouville integrals $J_{a+}^{\mu} f$ and $J_{b-}^{\mu} f$ of order $\alpha>0$ are defined by

$$
J_{a+}^{\mu} f(x)=\frac{1}{\Gamma(\mu)} \int_{a}^{x}(x-t)^{\mu-1} f(t) d t, \quad x>a
$$

and

$$
J_{b-}^{\mu} f(x)=\frac{1}{\Gamma(\mu)} \int_{x}^{b}(t-x)^{\mu-1} f(t) d t, \quad x<b
$$

respectively where $\Gamma(\mu)=\int_{0}^{\infty} e^{-t} u^{\mu-1} d u$. Here $J_{a+}^{0} f(x)=J_{b-}^{0} f(x)=f(x)$

In the case of $\mu=1$, the fractional integral reduces to classical integral.

The great impact of fractional calculus in pure and applied sciences can not be denied. Resultantly many researchers used the techniques of fractional calculus intensively to get the new refinements of the previously known results. For example, we refer the reader to [1-3] and references cited therein. In [12], Sarıkaya et. al. proved a new 
version of Hermite-Hadamard's inequalities in Riemann-Liouville fractional integral form as follows:

Theorem 2. Let $f:[a, b] \rightarrow \mathbb{R}$ be a positive function with $0 \leq a<b$ and $f \in$ $L_{1}[a, b]$. If $f$ is a convex function on $[a, b]$, then the following inequalities for fractional integrals holds:

$$
f\left(\frac{a+b}{2}\right) \leq \frac{\Gamma(\alpha+1)}{2(b-a)^{\alpha}}\left[J_{a+}^{\alpha} f(b)+J_{b-}^{\alpha} f(a)\right] \leq \frac{f(a)+f(b)}{2}
$$

with $\alpha>0$.

For further results related to Hermite-Hadamard type inequalities involving fractional integrals on can see [8,9,12-19].

In [5], Iscan et al. gave a generalization of (1.3) for harmonically convex functions as follows:

Theorem 3. Let $f: I \subset(0, \infty) \rightarrow \mathbb{R}$ be a function such that $f \in L[a, b]$, where $a, b \in I$ with $a<b$. If $f$ is a harmonically convex function on $[a, b]$, then the following inequalities for fractional integrals hold:

$$
\begin{aligned}
f\left(\frac{2 a b}{a+b}\right) & \leq \frac{\Gamma(\alpha+1)}{2}\left(\frac{a b}{b-a}\right)^{\alpha}\left\{J_{1 / a-}^{\alpha}(f \circ g)(1 / b)+J_{1 / b+}^{\alpha}(f \circ g)(1 / a)\right\} \\
& \leq \frac{f(a)+f(b)}{2}
\end{aligned}
$$

where $g(x)=1 / x$.

Katugampola gave a new fractional integral that generalizes the Riemann-Liouville and the Hadamard fractional integrals into a single form.

Definition 3 ([6]). Let $[a, b] \subset \mathbb{R}$ be a finite interval. Then, the left- and right-side Katugampola fractional integrals of order $(\alpha>0)$ of $f \in X_{c}^{p}(a, b)$ are defined:

$$
\rho_{l_{a+}^{\alpha}}^{\alpha} f(x)=\frac{\rho^{1-\alpha}}{\Gamma(\alpha)} \int_{a}^{x} \frac{t^{\rho-1}}{\left(x^{\rho}-t^{\rho}\right)^{1-\alpha}} f(t) d t
$$

and

$$
\rho \mathcal{d}_{b-}^{\alpha} f(x)=\frac{\rho^{1-\alpha}}{\Gamma(\alpha)} \int_{x}^{b} \frac{t^{\rho-1}}{\left(t^{\rho}-x^{\rho}\right)^{1-\alpha}} f(t) d t
$$

with $a<x<b$ and $\rho>0$, if the integral exist.

Theorem 4 ([6]). Let $\alpha>0$ and $\rho>0$. Then for $x>a$,

1. $\lim _{\rho \rightarrow 1} \rho \alpha_{a+}^{\alpha} f(x)=J_{a+}^{\alpha} f(x)$,

2. $\lim _{\rho \rightarrow 0^{+}}{ }^{\rho} d_{a+}^{\alpha} f(x)=H_{a+}^{\alpha} f(x)$.

Similar results also hold for right-sided operators.

The main purpose of this paper is to establish Hermite-Hadamard's inequalities for harmonically convex functions via Katugampola fractional integral. We also obtain Hermite-Hadamard type inequalities of these classes functions. 


\section{HERMITE-HADAMARD INEQUALITIES FOR HARMONICALLY CONVEXITY VIA KATUGAMPOLA FRACTIONAL INTEGRALS}

Consider the space $X_{c}^{p}(a, b)(c \in \mathbb{R}, 1 \leq p \leq \infty)$ consist of those complex-valued Lebesque measurable functions $\varphi$ on $(a, b)$ for which $\|\varphi\|_{X_{c}^{p}}<\infty$, with

$$
\|\varphi\|_{X_{c}^{p}}=\left(\int_{a}^{b}\left|x^{c} \varphi(x)\right|^{p} \frac{d x}{x}\right)^{1 / p} \quad(1 \leq p<\infty)
$$

and

$$
\|\varphi\|_{X_{c}^{p}}=\operatorname{esssup}_{x \in(a, b)}\left[x^{c}|\varphi(x)|\right] .
$$

Hermite-Hadamard's inequalities for harmonically convex functions can be represented in Katugampola fractional integral forms as follows:

Theorem 5. Let $\alpha>0$ and $\rho>0$. Let $f: I \subset(0, \infty) \rightarrow \mathbb{R}$ be a function such that $f \in X_{c}^{p}\left(a^{\rho}, b^{\rho}\right)$, where $a^{\rho}, b^{\rho} \in I$ with $a<b$. If $f$ is a harmonically convex function on $[a, b]$, then the following inequalities hold:

$$
\begin{aligned}
& f\left(\frac{2 a^{\rho} b^{\rho}}{a^{\rho}+b^{\rho}}\right) \\
\leq & \frac{\rho^{\alpha} \Gamma(\alpha+1)}{2}\left(\frac{a^{\rho} b^{\rho}}{b^{\rho}-a^{\rho}}\right)^{\alpha}\left\{{ }^{\rho}{\ell_{1 / a-}^{\alpha}}^{\alpha}(f \circ g)(1 / b)+{ }^{\rho}{\ell_{1 / b+}^{\alpha}}^{\alpha}(f \circ g)(1 / a)\right\} \\
\leq & \frac{f\left(a^{\rho}\right)+f\left(b^{\rho}\right)}{2} .
\end{aligned}
$$

where $g(x)=1 / x^{\rho}$.

Proof. Let $t \in[0,1]$. Consider $x, y \in[a, b], a \geq 0$, choosing $x^{\rho}=\frac{a^{\rho} b^{\rho}}{t^{\rho} b^{\rho}+\left(1-t^{\rho}\right) a^{\rho}}$, $y^{\rho}=\frac{a^{\rho} b^{\rho}}{t^{\rho} a^{\rho}+\left(1-t^{\rho}\right) b^{\rho}}$. Since $f$ is harmonically convex function on $[a, b]$, and from definition, we can write

$$
f\left(\frac{2 x^{\rho} y^{\rho}}{x^{\rho}+y^{\rho}}\right) \leq \frac{f\left(x^{\rho}\right)+f\left(y^{\rho}\right)}{2}
$$

Then we have

$$
f\left(\frac{2 a^{\rho} b^{\rho}}{a^{\rho}+b^{\rho}}\right) \leq \frac{f\left(\frac{a^{\rho} b^{\rho}}{t^{\rho} b^{\rho}+\left(1-t^{\rho}\right) a^{\rho}}\right)+f\left(\frac{a^{\rho} b^{\rho}}{t^{\rho} a^{\rho}+\left(1-t^{\rho}\right) b^{\rho}}\right)}{2}
$$

Multiplying both sides of (2.2) by $t^{\rho \alpha-1}$, then integrating the resulting inequality with respect to $t$ over $[0,1]$, we obtain

$$
f\left(\frac{2 a^{\rho} b^{\rho}}{a^{\rho}+b^{\rho}}\right)
$$




$$
\begin{aligned}
\leq & \frac{\rho \alpha}{2}\left\{\int_{0}^{1} t^{\rho \alpha-1} f\left(\frac{a^{\rho} b^{\rho}}{t^{\rho} b^{\rho}+\left(1-t^{\rho}\right) a^{\rho}}\right) d t+\int_{0}^{1} t^{\rho \alpha-1} f\left(\frac{a^{\rho} a^{\rho}}{t^{\rho} b^{\rho}+\left(1-t^{\rho}\right) b^{\rho}}\right) d t\right\} \\
& =\frac{\rho \alpha}{2}\left(\frac{a^{\rho} b^{\rho}}{b^{\rho}-a^{\rho}}\right)^{\alpha}\left\{\int_{1 / b}^{1 / a} \frac{x^{\rho-1}}{\left(x^{\rho}-\frac{1}{b^{\rho}}\right)^{1-\alpha}} f\left(\frac{1}{x^{\rho}}\right) d x\right. \\
& \left.+\int_{1 / a}^{1 / b} \frac{x^{\rho-1}}{\left(\frac{1}{a^{\rho}}-x^{\rho}\right)^{1-\alpha}} f\left(\frac{1}{x^{\rho}}\right) d x\right\} \\
& =\frac{\rho^{\alpha} \Gamma(\alpha+1)}{2}\left(\frac{a^{\rho} b^{\rho}}{b^{\rho}-a^{\rho}}\right)^{\alpha}\left\{{ }^{\rho} d_{1 / a-}^{\alpha}(f \circ g)(1 / b)+{ }^{\rho} d_{1 / b+}^{\alpha}(f \circ g)(1 / a)\right\}
\end{aligned}
$$

where $g(x)=1 / x^{\rho}$. So the first inequality is proved.

For the proof of the second inequality in (2.1), we first note that that for a harmonically convex function $f$, we have

$$
f\left(\frac{a^{\rho} b^{\rho}}{t^{\rho} b^{\rho}+\left(1-t^{\rho}\right) a^{\rho}}\right) \leq t^{\rho} f\left(a^{\rho}\right)+\left(1-t^{\rho}\right) b^{\rho}
$$

and

$$
f\left(\frac{a^{\rho} b^{\rho}}{t^{\rho} a^{\rho}+\left(1-t^{\rho}\right) b^{\rho}}\right) \leq t^{\rho} f\left(b^{\rho}\right)+\left(1-t^{\rho}\right) a^{\rho} .
$$

By adding these inequalities, we have

$$
f\left(\frac{a^{\rho} b^{\rho}}{t^{\rho} b^{\rho}+\left(1-t^{\rho}\right) a^{\rho}}\right)+f\left(\frac{a^{\rho} b^{\rho}}{t^{\rho} a^{\rho}+\left(1-t^{\rho}\right) b^{\rho}}\right) \leq f\left(a^{\rho}\right)+f\left(b^{\rho}\right) .
$$

Then multiplying both sides of (2.3) by $t^{\rho \alpha-1}$, and integrating the resulting inequality with respect to $t$ over $[0,1]$, we get

$$
\begin{aligned}
\int_{0}^{1} f\left(\frac{a^{\rho} b^{\rho}}{t^{\rho} b^{\rho}+\left(1-t^{\rho}\right) a^{\rho}}\right) t^{\rho \alpha-1} d t & +\int_{0}^{1} f\left(\frac{a^{\rho} b^{\rho}}{t^{\rho} a^{\rho}+\left(1-t^{\rho}\right) b^{\rho}}\right) t^{\rho \alpha-1} d t \\
& \leq[f(a)+f(b)] \int_{0}^{1} t^{\rho \alpha-1} d t
\end{aligned}
$$

i.e.

$$
\begin{aligned}
\frac{\rho^{\alpha} \Gamma(\alpha+1)}{2} & \left(\frac{a^{\rho} b^{\rho}}{b^{\rho}-a^{\rho}}\right)^{\alpha}\left\{\rho{ }^{\rho}{ }_{1 / a-}^{\alpha}(f \circ g)(1 / b)+{ }^{\rho} \ell_{1 / b+}^{\alpha}(f \circ g)(1 / a)\right\} \\
& \leq \frac{f\left(a^{\rho}\right)+f\left(b^{\rho}\right)}{2} .
\end{aligned}
$$

The proof is completed.

Remark 1. In Theorem 5, taking limit $\rho \rightarrow 1$ we obtain inequality of (1.4). 


\section{HERmite-HAdAmARd TyPE INEQUALITIES FOR KATUGAMPOLA FRACTIONAL INTEGRALS}

Let $f: I \subset(0, \infty) \rightarrow \mathbb{R}$ be a differentiable function on $I^{\circ}$, the interior of $I$, throughout this section we will take

$$
\begin{aligned}
I_{f}(g ; \alpha, a, b) & =\frac{f\left(a^{\rho}\right)+b^{\rho}}{2}-\frac{\rho^{\alpha} \Gamma(\alpha+1)}{2}\left(\frac{a^{\rho} b^{\rho}}{b^{\rho}-a^{\rho}}\right)^{\alpha} \\
& \left\{\rho_{d_{1 / a-}^{\alpha}}^{\alpha}(f \circ g)(1 / b)+{ }^{\rho} \mathcal{d}_{1 / b+}^{\alpha}(f \circ g)(1 / a)\right\},
\end{aligned}
$$

where $a^{\rho}, b^{\rho} \in I$ with $a<b . g(x)=1 / x^{\rho}$ and $\Gamma$ is Euler Gamma function.

Lemma 2. Let $\alpha>0$ and $\rho>0$. Let $f: I \subset(0, \infty) \rightarrow \mathbb{R}$ be a differentiable function such that $f \in X_{c}^{p}\left(a^{\rho}, b^{\rho}\right)$, where $a^{\rho}, b^{\rho} \in I$ with $a<b$. Then the following equality holds:

$I_{f}(g ; \alpha, a, b)=\frac{\rho a^{\rho} b^{\rho}\left(b^{\rho}-a^{\rho}\right)}{2} \int_{0}^{1} \frac{\left[t^{\rho \alpha}-\left(1-t^{\rho}\right)^{\alpha}\right] t^{\rho-1}}{\left[t^{\rho} a^{\rho}+\left(1-t^{\rho}\right) b^{\rho}\right]^{2}} f^{\prime}\left(\frac{a^{\rho} b^{\rho}}{t^{\rho} a^{\rho}+\left(1-t^{\rho}\right) b^{\rho}}\right) d t$.

Proof. Let $A_{t}=t^{\rho} a^{\rho}+\left(1-t^{\rho}\right) b^{\rho}$ and $B_{t}=t^{\rho} b^{\rho}+\left(1-t^{\rho}\right) a^{\rho}$. It suffices to note that

$$
\begin{aligned}
I_{f}(g ; \alpha, a, b) & =\frac{\rho a^{\rho} b^{\rho}\left(b^{\rho}-a^{\rho}\right)}{2} \int_{0}^{1} \frac{\left[t^{\rho \alpha}-\left(1-t^{\rho}\right)^{\alpha}\right] t^{\rho-1}}{\left[t^{\rho} a^{\rho}+\left(1-t^{\rho}\right) b^{\rho}\right]^{2}} f^{\prime}\left(\frac{a^{\rho} b^{\rho}}{t^{\rho} a^{\rho}+\left(1-t^{\rho}\right) b^{\rho}}\right) d t \\
& =\frac{\rho a^{\rho} b^{\rho}\left(b^{\rho}-a^{\rho}\right)}{2} \int_{0}^{1} \frac{t^{\rho \alpha} t^{\rho-1}}{A_{t}^{2}} f^{\prime}\left(\frac{a^{\rho} b^{\rho}}{A_{t}}\right) d t \\
& -\frac{\rho a^{\rho} b^{\rho}\left(b^{\rho}-a^{\rho}\right)}{2} \int_{0}^{1} \frac{\left(1-t^{\rho}\right)^{\alpha} t^{\rho-1}}{A_{t}^{2}} f^{\prime}\left(\frac{a^{\rho} b^{\rho}}{A_{t}}\right) d t \\
& =I_{1}+I_{2}
\end{aligned}
$$

By integrating by part, we get

$$
\begin{aligned}
I_{1} & =\frac{1}{2}\left[\left.t^{\rho \alpha} f\left(\frac{a^{\rho} b^{\rho}}{A_{t}}\right)\right|_{0} ^{1}-\rho \alpha \int_{0}^{1} t^{\rho \alpha-1} f\left(\frac{a^{\rho} b^{\rho}}{A_{t}}\right) d t\right] \\
& =\frac{1}{2}\left[f\left(b^{\rho}\right)-\rho \alpha\left(\frac{a^{\rho} b^{\rho}}{b^{\rho}-a^{\rho}}\right)^{\alpha} \int_{1 / b}^{1 / a} \frac{x^{\rho-1}}{\left(x^{\rho}-\frac{1}{b^{\rho}}\right)^{1-\alpha}} f\left(\frac{1}{x^{\rho}}\right) d x\right] \\
& =\frac{1}{2}\left[f\left(b^{\rho}\right)-\rho^{\alpha} \Gamma(\alpha+1)\left(\frac{a^{\rho} b^{\rho}}{b^{\rho}-a^{\rho}}\right)^{\alpha}{ }^{\alpha} d_{1 / a-}^{\alpha}(f \circ g)(1 / b)\right]
\end{aligned}
$$


and similarly we get

$$
\begin{aligned}
I_{2} & =-\frac{1}{2}\left[\left.\left(1-t^{\rho}\right)^{\alpha} f\left(\frac{a^{\rho} b^{\rho}}{A_{t}}\right)\right|_{0} ^{1}+\rho \alpha \int_{0}^{1}\left(1-t^{\rho}\right)^{\alpha-1} t^{\rho-1} f\left(\frac{a^{\rho} b^{\rho}}{A_{t}}\right) d t\right] \\
& =\frac{1}{2}\left[f\left(a^{\rho}\right)-\rho \alpha \int_{0}^{1} u^{\rho \alpha-1} f\left(\frac{a^{\rho} b^{\rho}}{B_{t}}\right) d u\right] \\
& =\frac{1}{2}\left[f\left(a^{\rho}\right)-\rho \alpha\left(\frac{a^{\rho} b^{\rho}}{b^{\rho}-a^{\rho}}\right)^{\alpha} \int_{1 / a}^{1 / b} \frac{x^{\rho-1}}{\left(\frac{1}{a^{\rho}}-x^{\rho}\right)^{1-\alpha}} f\left(\frac{1}{x^{\rho}}\right) d x\right] \\
& =\frac{1}{2}\left[f\left(a^{\rho}\right)-\rho^{\alpha} \Gamma(\alpha+1)\left(\frac{a^{\rho} b^{\rho}}{b^{\rho}-a^{\rho}}\right)^{\alpha} d_{1 / b+}^{\alpha}(f \circ g)(1 / a)\right]
\end{aligned}
$$

Using (3.3) and (3.4) in (3.2), we get equality (3.1).

Remark 2. In Lemma 2, taking limit $\rho \rightarrow 1$ we obtain inequality Lemma 3 in [5].

Theorem 6. Let $\alpha>0$ and $\rho>0$. Let $f: I \subset(0, \infty) \rightarrow \mathbb{R}$ be a differentiable function such that $f \in X_{c}^{p}\left(a^{\rho}, b^{\rho}\right)$, where $a^{\rho}, b^{\rho} \in I$ with $a<b$. If $|f|^{q}$ is a harmonically convex function on $[a, b]$ for some fixed $q \geq 1$, then the following inequalities holds:

$$
\begin{aligned}
&\left|I_{f}(g ; \alpha, a, b)\right| \leq \frac{\rho a^{\rho} b^{\rho}\left(b^{\rho}-a^{\rho}\right)}{2} \Lambda_{1}^{1-1 / q}(\alpha ; a, b) \\
&\left(\Lambda_{2}(\alpha ; a, b)\left|f^{\prime}(b)\right|^{q}+\Lambda_{3}(\alpha ; a, b)\left|f^{\prime}(a)\right|^{q}\right)^{1 / q}
\end{aligned}
$$

where

$$
\begin{aligned}
\Lambda_{1}(\alpha ; a, b)= & \frac{b^{-2 \rho}}{\rho(\alpha+1)}\left[{ }_{2} F_{1}\left(2, \alpha+1 ; \alpha+2 ; 1-\frac{a^{\rho}}{b^{\rho}}\right)\right. \\
& \left.+{ }_{2} F_{1}\left(2,1 ; \alpha+2 ; 1-\frac{a^{\rho}}{b^{\rho}}\right)\right], \\
\Lambda_{2}(\alpha ; a, b)= & \frac{b^{-2 \rho}}{\rho(\alpha+2)}\left[{ }_{2} F_{1}\left(2, \alpha+2 ; \alpha+3 ; 1-\frac{a^{\rho}}{b^{\rho}}\right)\right. \\
& \left.+\frac{1}{\alpha+1}{ }_{2} F_{1}\left(2,2 ; \alpha+3 ; 1-\frac{a^{\rho}}{b^{\rho}}\right)\right], \\
\Lambda_{3}(\alpha ; a, b)= & \frac{b^{-2 \rho}}{\rho(\alpha+2)}\left[\frac{1}{\alpha+1}{ }_{2} F_{1}\left(2, \alpha+1 ; \alpha+3 ; 1-\frac{a^{\rho}}{b^{\rho}}\right)\right. \\
& \left.+{ }_{2} F_{1}\left(2,1 ; \alpha+3 ; 1-\frac{a^{\rho}}{b^{\rho}}\right)\right] .
\end{aligned}
$$

Proof. Let $A_{t}=t^{\rho} a^{\rho}+\left(1-t^{\rho}\right) b^{\rho}$. From Lemma 2, using the property of the modulus, the power mean inequality and the harmonically convexity of $|f|^{q}$, we get

$\left|I_{f}(g ; \alpha, a, b)\right|$ 


$$
\begin{aligned}
\leq & \frac{\rho a^{\rho} b^{\rho}\left(b^{\rho}-a^{\rho}\right)}{2} \int_{0}^{1} \frac{\left|t^{\rho \alpha}-\left(1-t^{\rho}\right)^{\alpha}\right|\left|t^{\rho-1}\right|}{A_{t}^{2}}\left|f^{\prime}\left(\frac{a^{\rho} b^{\rho}}{A_{t}}\right)\right| d t \\
\leq & \frac{\rho a^{\rho} b^{\rho}\left(b^{\rho}-a^{\rho}\right)}{2}\left(\int_{0}^{1} \frac{\left|t^{\rho \alpha}-\left(1-t^{\rho}\right)^{\alpha}\right|\left|t^{\rho-1}\right|}{A_{t}^{2}} d t\right)^{1-1 / q} \\
& \times\left(\int_{0}^{1} \frac{\left|t^{\rho \alpha}-\left(1-t^{\rho}\right)^{\alpha}\right|\left|t^{\rho-1}\right|}{A_{t}^{2}}\left|f^{\prime}\left(\frac{a^{\rho} b^{\rho}}{A_{t}}\right)\right| d t\right)^{1 / q} \\
\leq & \frac{\rho a^{\rho} b^{\rho}\left(b^{\rho}-a^{\rho}\right)}{2}\left(\int_{0}^{1} \frac{\left|t^{\rho \alpha}+\left(1-t^{\rho}\right)^{\alpha}\right|\left|t^{\rho-1}\right|}{A_{t}^{2}} d t\right)^{1-1 / q} \\
& \times\left(\int_{0}^{1} \frac{\left|t^{\rho \alpha}+\left(1-t^{\rho}\right)^{\alpha}\right|\left|t^{\rho-1}\right|}{A_{t}^{2}}\left(t^{\rho}\left|f^{\prime}\left(b^{\rho}\right)\right|^{q}+\left(1-t^{\rho}\right)\left|f^{\prime}\left(a^{\rho}\right)\right|^{q}\right) d t\right)^{1 / q} \\
\leq & \frac{\rho a^{\rho} b^{\rho}\left(b^{\rho}-a^{\rho}\right)}{2} \Lambda_{1}^{1-1 / q}(\alpha ; a, b)\left(\Lambda_{2}(\alpha ; a, b)\left|f^{\prime}(b)\right|^{q}+\Lambda_{3}(\alpha ; a, b)\left|f^{\prime}(a)\right|^{q}\right)^{1 / q} .
\end{aligned}
$$

Calculating $\Lambda_{1}(\alpha ; a, b), \Lambda_{2}(\alpha ; a, b)$ and $\Lambda_{3}(\alpha ; a, b)$, we have

$$
\begin{aligned}
& \Lambda_{1}(\alpha ; a, b) \\
& =\int_{0}^{1} \frac{\left[t^{\rho \alpha}+\left(1-t^{\rho}\right)^{\alpha}\right] t^{\rho-1}}{A_{t}^{2}} d t \\
& =b^{-2 \rho} \int_{0}^{1}\left(u^{\alpha}+(1-u)^{\alpha}\right)\left(1-\left(1-\frac{a^{\rho}}{b^{\rho}}\right) u\right)^{-2} d t \\
& =\frac{b^{-2 \rho}}{\rho(\alpha+1)}\left[{ }_{2} F_{1}\left(2, \alpha+1 ; \alpha+2 ; 1-\frac{a^{\rho}}{b^{\rho}}\right)+{ }_{2} F_{1}\left(2,1 ; \alpha+2 ; 1-\frac{a^{\rho}}{b^{\rho}}\right)\right] .
\end{aligned}
$$

Similarly, we get

$$
\begin{aligned}
& \Lambda_{2}(\alpha ; a, b) \\
& =\int_{0}^{1} \frac{\left[t^{\rho \alpha}+\left(1-t^{\rho}\right)^{\alpha}\right] t^{\rho-1}}{A_{t}^{2}} t^{\rho} d t \\
& =\frac{b^{-2 \rho}}{\rho(\alpha+2)}\left[{ }_{2} F_{1}\left(2, \alpha+2 ; \alpha+3 ; 1-\frac{a^{\rho}}{b^{\rho}}\right)+\frac{1}{\alpha+1}{ }_{2} F_{1}\left(2,2 ; \alpha+3 ; 1-\frac{a^{\rho}}{b^{\rho}}\right)\right]
\end{aligned}
$$

and

$$
\begin{aligned}
& \Lambda_{3}(\alpha ; a, b) \\
& =\int_{0}^{1} \frac{\left[t^{\rho \alpha}+\left(1-t^{\rho}\right)^{\alpha}\right] t^{\rho-1}}{A_{t}^{2}} t^{\rho} d t
\end{aligned}
$$




$$
=\frac{b^{-2 \rho}}{\rho(\alpha+2)}\left[\frac{1}{\alpha+1}{ }_{2} F_{1}\left(2, \alpha+1 ; \alpha+3 ; 1-\frac{a^{\rho}}{b^{\rho}}\right)+{ }_{2} F_{1}\left(2,1 ; \alpha+3 ; 1-\frac{a^{\rho}}{b^{\rho}}\right)\right]
$$

So, if we use (3.7)-(3.9) in (3.6), we obtain the inequality of (3.5). This completes the proof.

Remark 3. In Theorem 6, taking limit $\rho \rightarrow 1$ we obtain Theorem 5 in [5].

Theorem 7. Let $\alpha>0$ and $\rho>0$. Let $f: I \subset(0, \infty) \rightarrow \mathbb{R}$ be a differentiable function such that $f \in X_{c}^{p}\left(a^{\rho}, b^{\rho}\right)$, where $a^{\rho}, b^{\rho} \in I$ with $a<b$. If $|f|^{l}$ is a harmonically convex function on $[a, b]$ for some fixed $l \geq 1$, then the following inequalities holds:

$$
\begin{aligned}
\left|I_{f}(g ; \alpha, a, b)\right| & \leq \frac{\rho a^{\rho} b^{\rho}\left(b^{\rho}-a^{\rho}\right)}{2} \Lambda_{4}^{1-1 / q}(\alpha ; a, b) \\
& \left(\Lambda_{5}(\alpha ; a, b)\left|f^{\prime}(b)\right|^{q}+\Lambda_{6}(\alpha ; a, b)\left|f^{\prime}(a)\right|^{q}\right)^{1 / q}
\end{aligned}
$$

where

$$
\begin{aligned}
\Lambda_{4} & =\frac{b^{-2 \rho}}{\rho(\alpha+1)}\left[{ }_{2} F_{1}\left(2, \alpha+1 ; \alpha+2 ; 1-\frac{a^{\rho}}{b^{\rho}}\right)-{ }_{2} F_{1}\left(2,1 ; \alpha+2 ; 1-\frac{a^{\rho}}{b^{\rho}}\right)\right. \\
& +{ }_{2} F_{1}\left(2,1 ; \alpha+2 ; \frac{1}{2}\left(1-\frac{a^{\rho}}{b^{\rho}}\right)\right) \\
\Lambda_{5} & =\frac{b^{-2 \rho}}{\rho(\alpha+2)}\left[{ }_{2} F_{1}\left(2, \alpha+2 ; \alpha+3 ; 1-\frac{a^{\rho}}{b^{\rho}}\right)-\frac{1}{\alpha+1}{ }_{2} F_{1}\left(2,2 ; \alpha+3 ; 1-\frac{a^{\rho}}{b^{\rho}}\right)\right. \\
& +\frac{1}{2(\alpha+1)} 2 F_{1}\left(2,2 ; \alpha+3 ; \frac{1}{2}\left(1-\frac{a^{\rho}}{b^{\rho}}\right)\right) \\
\Lambda_{6} & =\frac{b^{-2 \rho}}{\rho(\alpha+2)}\left[\frac{1}{\alpha+1}{ }_{2} F_{1}\left(2, \alpha+1 ; \alpha+3 ; 1-\frac{a^{\rho}}{b^{\rho}}\right)-{ }_{2} F_{1}\left(2,1 ; \alpha+3 ; 1-\frac{a^{\rho}}{b^{\rho}}\right)\right. \\
& +{ }_{2} F_{1}\left(2,1 ; \alpha+3 ; \frac{1}{2}\left(1-\frac{a^{\rho}}{b^{\rho}}\right)\right) .
\end{aligned}
$$

Proof. Let $A_{t}=t^{\rho} a^{\rho}+\left(1-t^{\rho}\right) b^{\rho}$. From Lemma 2, using the property of the modulus, the power mean inequality and the harmonically convexity of $|f|^{q}$, we have

$$
\begin{aligned}
& \left|I_{f}(g ; \alpha, a, b)\right| \\
\leq & \frac{\rho a^{\rho} b^{\rho}\left(b^{\rho}-a^{\rho}\right)}{2} \int_{0}^{1} \frac{\left|t^{\rho \alpha}-\left(1-t^{\rho}\right)^{\alpha}\right|\left|t^{\rho-1}\right|}{A_{t}^{2}}\left|f^{\prime}\left(\frac{a^{\rho} b^{\rho}}{A_{t}}\right)\right| d t \\
\leq & \frac{\rho a^{\rho} b^{\rho}\left(b^{\rho}-a^{\rho}\right)}{2}\left(\int_{0}^{1} \frac{\left|t^{\rho \alpha}-\left(1-t^{\rho}\right)^{\alpha}\right|\left|t^{\rho-1}\right|}{A_{t}^{2}} d t\right)^{1-1 / q}
\end{aligned}
$$




$$
\begin{aligned}
& \times\left(\int_{0}^{1} \frac{\left|t^{\rho \alpha}-\left(1-t^{\rho}\right)^{\alpha}\right|\left|t^{\rho-1}\right|}{A_{t}^{2}}\left|f^{\prime}\left(\frac{a^{\rho} b^{\rho}}{A_{t}}\right)\right| d t\right)^{1 / q} \\
\leq & \frac{\rho a^{\rho} b^{\rho}\left(b^{\rho}-a^{\rho}\right)}{2}\left(\int_{0}^{1} \frac{\left|t^{\rho \alpha}-\left(1-t^{\rho}\right)^{\alpha}\right|\left|t^{\rho-1}\right|}{A_{t}^{2}} d t\right)^{1-1 / q} \\
& \times\left(\int_{0}^{1} \frac{\left|t^{\rho \alpha}-\left(1-t^{\rho}\right)^{\alpha}\right|\left|t^{\rho-1}\right|}{A_{t}^{2}}\left(t^{\rho}\left|f^{\prime}\left(b^{\rho}\right)\right|^{q}+\left(1-t^{\rho}\right)\left|f^{\prime}\left(a^{\rho}\right)\right|^{q}\right) d t\right)^{1 / q} \\
\leq & \frac{\rho a^{\rho} b^{\rho}\left(b^{\rho}-a^{\rho}\right)}{2} K_{1}^{1-1 / q}(\alpha ; a, b)\left(K_{2}(\alpha ; a, b)\left|f^{\prime}(b)\right|^{q}+K_{3}(\alpha ; a, b)\left|f^{\prime}(a)\right|^{q}\right)^{1 / q} .
\end{aligned}
$$

Calculating $K_{1}, K_{2}$ and $K_{3}$, by Lemma 1 , we get

$$
\begin{aligned}
K_{1} & =\int_{0}^{1} \frac{\left|t^{\rho \alpha}-\left(1-t^{\rho}\right)^{\alpha}\right|\left|t^{\rho-1}\right|}{A_{t}^{2}} d t \\
& =\int_{0}^{1 / 2} \frac{\left(\left(1-t^{\rho}\right)^{\alpha}-t^{\rho \alpha}\right) t^{\rho-1}}{A_{t}^{2}} d t+\int_{1 / 2}^{1} \frac{\left(t^{\rho \alpha}-\left(1-t^{\rho}\right)^{\alpha}\right) t^{\rho-1}}{A_{t}^{2}} d t \\
& =\int_{0}^{1} \frac{\left(t^{\rho \alpha}-\left(1-t^{\rho}\right)^{\alpha}\right) t^{\rho-1}}{A_{t}^{2}} d t+2 \int_{0}^{1 / 2} \frac{\left(\left(1-t^{\rho}\right)^{\alpha}-t^{\rho \alpha}\right) t^{\rho-1}}{A_{t}^{2}} d t \\
\leq & \int_{0}^{1} u^{\alpha} A_{u}^{-2} d u-\int_{0}^{1}(1-u)^{\alpha} A_{u}^{-2} d u+2 \int_{0}^{1 / 2}(1-2 u)^{\alpha} A_{u}^{-2} d u \\
& =\frac{b^{-2 \rho}}{\rho(\alpha+1)}\left[{ }_{2} F_{1}\left(2, \alpha+1 ; \alpha+2 ; 1-\frac{a^{\rho}}{b^{\rho}}\right)-{ }_{2} F_{1}\left(2,1 ; \alpha+2 ; 1-\frac{a^{\rho}}{b^{\rho}}\right)\right. \\
& +{ }_{2} F_{1}\left(2,1 ; \alpha+2 ; \frac{1}{2}\left(1-\frac{a^{\rho}}{b^{\rho}}\right)\right) .
\end{aligned}
$$

and similarly we obtain

$$
\begin{aligned}
K_{2} & =\int_{0}^{1} \frac{\left|t^{\rho \alpha}-\left(1-t^{\rho}\right)^{\alpha}\right|\left|t^{\rho-1}\right|}{A_{t}^{2}} t^{\rho} d t \\
\leq & \int_{0}^{1} u^{\alpha+1} A_{u}^{-2} d u-\int_{0}^{1}(1-u)^{\alpha} u A_{u}^{-2} d u+2 \int_{0}^{1 / 2}(1-2 u)^{\alpha} u A_{u}^{-2} d u \\
& =\frac{b^{-2 \rho}}{\rho(\alpha+2)}\left[{ }_{2} F_{1}\left(2, \alpha+2 ; \alpha+3 ; 1-\frac{a^{\rho}}{b^{\rho}}\right)-\frac{1}{\alpha+1}{ }_{2} F_{1}\left(2,2 ; \alpha+3 ; 1-\frac{a^{\rho}}{b^{\rho}}\right)\right. \\
& +\frac{1}{2(\alpha+1)}{ }_{2} F_{1}\left(2,2 ; \alpha+3 ; \frac{1}{2}\left(1-\frac{a^{\rho}}{b^{\rho}}\right)\right),
\end{aligned}
$$


and

$$
\begin{aligned}
& K_{3}=\int_{0}^{1} \frac{\left|t^{\rho \alpha}-\left(1-t^{\rho}\right)^{\alpha}\right|\left|t^{\rho-1}\right|}{A_{t}^{2}}(1-t)^{\rho} d t \\
\leq & \int_{0}^{1} t^{\alpha}(1-u) A_{u}^{-2} d u-\int_{0}^{1}(1-u)^{\alpha+1} A_{u}^{-2} d u+2 \int_{0}^{1 / 2}(1-2 u)^{\alpha}(1-u) A_{u}^{-2} d u \\
& =\frac{b^{-2 \rho}}{\rho(\alpha+2)}\left[\frac{1}{\alpha+1}{ }_{2} F_{1}\left(2, \alpha+1 ; \alpha+3 ; 1-\frac{a^{\rho}}{b^{\rho}}\right)-{ }_{2} F_{1}\left(2,1 ; \alpha+3 ; 1-\frac{a^{\rho}}{b^{\rho}}\right)\right. \\
& +{ }_{2} F_{1}\left(2,1 ; \alpha+3 ; \frac{1}{2}\left(1-\frac{a^{\rho}}{b^{\rho}}\right)\right) .
\end{aligned}
$$

So, if we use (3.12)-(3.14) in (3.11), we get the inequality (3.10).

Remark 4. In Theorem 7, taking limit $\rho \rightarrow 1$ we obtain Theorem 6 in [5].

Theorem 8. Let $\alpha>0$ and $\rho>0$. Let $f: I \subset(0, \infty) \rightarrow \mathbb{R}$ be a differentiable function such that $f \in X_{c}^{p}\left(a^{\rho}, b^{\rho}\right)$, where $a^{\rho}, b^{\rho} \in I$ with $a<b$. If $|f|^{l}$ is a harmonically convex function on $[a, b]$ for some fixed $l>1,1 / k+1 / l=1$, then the following inequalities holds:

$$
\left|I_{f}(g ; \alpha, a, b)\right|=\frac{a^{\rho}\left(b^{\rho}-a^{\rho}\right)}{2 b^{\rho}}\left(\Lambda_{7}^{1 / k}+\Lambda_{8}^{1 / k}\right)\left(\frac{\left|f^{\prime}\left(a^{\rho}\right)\right|^{l}+\left|f^{\prime}\left(b^{\rho}\right)\right|^{l}}{\rho+1}\right)^{1 / l}
$$

where

$$
\begin{aligned}
& \Lambda_{7}=B\left(\frac{\rho k-k+1}{p}, \alpha k+1\right){ }_{2} F_{1}\left(2 k, \frac{\rho k-k+1}{\rho} ; \alpha k+k+1+\frac{1-k}{\rho} ; 1-\frac{a^{\rho}}{b^{\rho}}\right) \\
& \Lambda_{8}=\frac{1}{\left(\alpha k+k+\frac{1-k}{\rho}\right)}{ }_{2} F_{1}\left(2 k, \alpha k+k+\frac{1-k}{\rho} ; \alpha k+k+1+\frac{1-k}{\rho} ; 1-\frac{a^{\rho}}{b^{\rho}}\right)
\end{aligned}
$$

Proof. Let $A_{t}=t^{\rho} a^{\rho}+\left(1-t^{\rho}\right) b^{\rho}$. From Lemma 2, Hölder inequality and the harmonically convexity of $|f|^{q}$, we have

$$
\begin{aligned}
& \left|I_{f}(g ; \alpha, a, b)\right| \\
\leq & \frac{a^{\rho} b^{\rho}\left(b^{\rho}-a^{\rho}\right)}{2}\left[\int_{0}^{1} \frac{t^{\rho \alpha} t^{\rho-1}}{A_{t}^{2}}\left|f^{\prime}\left(\frac{a^{\rho} b^{\rho}}{A_{t}}\right)\right| d t+\int_{0}^{1} \frac{\left(1-t^{\rho}\right)^{\alpha} t^{\rho-1}}{A_{t}^{2}}\left|f^{\prime}\left(\frac{a^{\rho} b^{\rho}}{A_{t}}\right)\right| d t\right] \\
\leq & \frac{a^{\rho} b^{\rho}\left(b^{\rho}-a^{\rho}\right)}{2}\left\{\left(\int_{0}^{1} \frac{t^{\rho \alpha k} t^{k(\rho-1)}}{A_{t}^{2 k}} d t\right)^{1 / k}\left(\int_{0}^{1}\left|f^{\prime}\left(\frac{a^{\rho} b^{\rho}}{A_{t}^{2 k}}\right)\right|^{l}\right)^{1 / l}\right. \\
& \left.+\left(\int_{0}^{1} \frac{\left(1-t^{\rho}\right)^{\alpha k} t^{k(\rho-1)}}{A_{t}^{2 k}} d t\right)^{1 / k}\left(\int_{0}^{1}\left|f^{\prime}\left(\frac{a^{\rho} b^{\rho}}{A_{t}^{2 k}}\right)\right|^{l}\right)^{1 / l}\right\}
\end{aligned}
$$




$$
\begin{aligned}
& \leq \frac{a^{\rho} b^{\rho}\left(b^{\rho}-a^{\rho}\right)}{2}\left(K_{4}^{1 / k}+K_{5}^{1 / k}\right)\left(\int_{0}^{1}\left[t^{\rho}\left|f^{\prime}\left(b^{\rho}\right)\right|^{l}+\left(1-t^{\rho}\right)\left|f^{\prime}\left(a^{\rho}\right)\right|^{l}\right] d t\right)^{1 / l} \\
& \leq \frac{a^{\rho} b^{\rho}\left(b^{\rho}-a^{\rho}\right)}{2}\left(K_{4}^{1 / k}+K_{5}^{1 / k}\right)\left(\frac{\left|f^{\prime}\left(a^{\rho}\right)\right|^{l}+\left|f^{\prime}\left(b^{\rho}\right)\right|^{l}}{\rho+1}\right)^{1 / l}
\end{aligned}
$$

Calculating $K_{4}$ and $K_{5}$, we obtain

$$
\begin{aligned}
& K_{4}=\int_{0}^{1} \frac{\left(1-t^{\rho}\right)^{\alpha k} t^{k(\rho-1)}}{A_{t}^{2 k}} d t \\
& =\frac{b^{-2 \rho k}}{B\left(\frac{\rho k-k+1}{p}, \alpha k+1\right)}{ }_{2} F_{1}\left(2 k, \frac{\rho k-k+1}{\rho} ; \alpha k+k+1+\frac{1-k}{\rho} ; 1-\frac{a^{\rho}}{b^{\rho}}\right) \\
& K_{5}=\int_{0}^{1} \frac{t^{\rho \alpha k} t^{k(\rho-1)}}{A_{t}^{2 k}} d t \\
& =\left(\alpha k+k+\frac{1-k}{\rho}\right) b^{-2 \rho k}{ }_{2} F_{1}\left(2 k, \alpha k+k+\frac{1-k}{\rho} ; \alpha k+k+1+\frac{1-k}{\rho} ; 1-\frac{a^{\rho}}{b^{\rho}}\right)
\end{aligned}
$$

So, if we use (3.17) and (3.18) in (3.16), we get the inequality of (3.15). This completes the proof.

Remark 5. In Theorem 8, taking limit $\rho \rightarrow 1$ we obtain Theorem 7 in [5].

Theorem 9. Let $\alpha>0$ and $\rho>0$. Let $f: I \subset(0, \infty) \rightarrow \mathbb{R}$ be a differentiable function such that $f \in X_{c}^{p}\left(a^{\rho}, b^{\rho}\right)$, where $a^{\rho}, b^{\rho} \in I$ with $a<b$. If $|f|^{l}$ is a harmonically convex function on $[a, b]$ for some fixed $l>1,1 / k+1 / l=1$, then the following inequalities holds:

$$
\left|I_{f}(g ; \alpha, a, b)\right| \leq \frac{\rho a^{\rho} b^{\rho}\left(b^{\rho}-a^{\rho}\right)}{2} \Lambda_{9}^{1 / k}\left(\Lambda_{10}\left|f^{\prime}\left(b^{\rho}\right)\right|^{l}+\Lambda_{11}\left|f^{\prime}\left(a^{\rho}\right)\right|^{l}\right)^{1 / l}
$$

where

$$
\begin{aligned}
\Lambda_{9} & =b^{-2 \rho k}{ }_{2} F_{1}\left(2 k, \frac{1}{\rho} ; \frac{\rho+1}{\rho} ; 1-\frac{a^{\rho}}{b^{\rho}}\right) \\
\Lambda_{10} & =\frac{1}{\rho 2^{\frac{\rho+1}{\rho}}} B\left(\frac{\rho+1}{\rho}, \alpha l+1\right)+\frac{\alpha l+1}{2 \rho}{ }_{2} F_{1}\left(\frac{-1}{\rho}, 1 ; \alpha l+2 ; \frac{1}{2}\right) \\
\Lambda_{11} & =\frac{1}{\rho 2^{\frac{1}{\rho}}} B\left(\frac{1}{\rho}, \alpha l+1\right){ }_{2} F_{1}\left(-1, \frac{1}{\rho} ; \alpha l+\frac{1}{\rho}+1 ; \frac{1}{2}\right) \\
& +\frac{(\alpha l+1)(\alpha l+2)}{\rho 2^{\frac{2-\rho}{\rho}}}{ }_{2} F_{1}\left(\frac{1-\rho}{\rho}, 2 ; \alpha l+3 ; \frac{1}{2}\right) .
\end{aligned}
$$


Proof. Let $A_{t}=t^{\rho} a^{\rho}+\left(1-t^{\rho}\right) b^{\rho}$. From Lemma 1, Lemma 2, Hölder inequality and the harmonically convexity of $|f|^{q}$, we have

$$
\begin{aligned}
\left|I_{f}(g ; \alpha, a, b)\right| \leq & \frac{\rho a^{\rho} b^{\rho}\left(b^{\rho}-a^{\rho}\right)}{2} \int_{0}^{1} \frac{\left|t^{\rho \alpha}-\left(1-t^{\rho}\right)^{\alpha}\right|\left|t^{\rho-1}\right|}{A_{t}^{2}}\left|f^{\prime}\left(\frac{a^{\rho} b^{\rho}}{A_{t}}\right)\right| d t \\
\leq & \frac{\rho a^{\rho} b^{\rho}\left(b^{\rho}-a^{\rho}\right)}{2}\left(\int_{0}^{1} \frac{1}{A_{t}^{2 k}} d t\right)^{1 / k} \\
& \times\left(\int_{0}^{1}\left|t^{\rho \alpha}-\left(1-t^{\rho}\right)^{\alpha}\right|^{l}\left|t^{\rho-1}\right|^{l}\left|f^{\prime}\left(\frac{a^{\rho} b^{\rho}}{A_{t}}\right)\right|^{l} d t\right)^{1 / l} \\
\leq & \frac{\rho a^{\rho} b^{\rho}\left(b^{\rho}-a^{\rho}\right)}{2}\left(\int_{0}^{1} \frac{1}{A_{t}^{2 k}} d t\right)^{1 / k} \\
& \times\left(\int_{0}^{1}\left|1-2 t^{\rho}\right|^{\alpha l}\left[t^{\rho}\left|f^{\prime}\left(b^{\rho}\right)\right|^{l}+\left(1-t^{\rho}\right)\left|f^{\prime}\left(a^{\rho}\right)\right|^{l}\right] d t\right)^{1 / l} \\
\leq & \frac{\rho a^{\rho} b^{\rho}\left(b^{\rho}-a^{\rho}\right)}{2} K_{6}^{1 / k}\left(K_{7}\left|f^{\prime}\left(b^{\rho}\right)\right|^{l}+K_{8}\left|f^{\prime}\left(a^{\rho}\right)\right|^{l}\right)^{1 / l} .
\end{aligned}
$$

where

$$
\begin{aligned}
& \Lambda_{9}=\int_{0}^{1} \frac{1}{A_{t}^{2 k}} d t=b^{-2 \rho k}{ }_{2} F_{1}\left(2 k, \frac{1}{\rho} ; \frac{\rho+1}{\rho} ; 1-\frac{a^{\rho}}{b^{\rho}}\right) \\
& \Lambda_{10}=\int_{0}^{1}\left|1-2 t^{\rho}\right|^{\alpha l} t^{\rho} d t \\
& =\int_{0}^{1 / 2^{1 / \rho}}\left(1-2 t^{\rho}\right)^{\alpha l} t^{\rho} d t+\int_{1 / 2^{1 / \rho}}^{1}\left(2 t^{\rho}-1\right)^{\alpha l} t^{\rho} d t \\
& =\frac{1}{\rho 2^{\frac{\rho+1}{\rho}}} B\left(\frac{\rho+1}{\rho}, \alpha l+1\right)+\frac{\alpha l+1}{2 \rho}{ }_{2} F_{1}\left(\frac{-1}{\rho}, 1 ; \alpha l+2 ; \frac{1}{2}\right) \\
& \Lambda_{11}=\int_{0}^{1}\left|1-2 t^{\rho}\right|^{\alpha l}\left(1-t^{\rho}\right) d t \\
& =\int_{0}^{1 / 2^{1 / \rho}}\left(1-2 t^{\rho}\right)^{\alpha l}\left(1-t^{\rho}\right) d t+\int_{1 / 2^{1 / \rho}}^{1}\left(2 t^{\rho}-1\right)^{\alpha l}\left(1-t^{\rho}\right) d t \\
& =\frac{1}{\rho 2^{\frac{1}{\rho}}} B\left(\frac{1}{\rho}, \alpha l+1\right){ }_{2} F_{1}\left(-1, \frac{1}{\rho} ; \alpha l+\frac{1}{\rho}+1 ; \frac{1}{2}\right) \\
& +\frac{(\alpha l+1)(\alpha l+2)}{\rho 2^{\frac{2-\rho}{\rho}}}{ }_{2} F_{1}\left(\frac{1-\rho}{\rho}, 2 ; \alpha l+3 ; \frac{1}{2}\right) \text {. }
\end{aligned}
$$

So, if we use (3.24)-(3.26) in (3.23), we get desired result. 
Remark 6. In Theorem 9, taking limit $\rho \rightarrow 1$ we obtain Theorem 8 in [5].

Theorem 10. Let $\alpha>0$ and $\rho>0$. Let $f: I \subset(0, \infty) \rightarrow \mathbb{R}$ be a differentiable function such that $f \in X_{c}^{p}\left(a^{\rho}, b^{\rho}\right)$, where $a^{\rho}, b^{\rho} \in I$ with $a<b$. If $|f|^{q}$ is a harmonically convex function on $[a, b]$ for some fixed $q>1,1 / k+1 / l=1$, then the following inequalities holds:

$$
\left|I_{f}(g ; \alpha, a, b)\right|=\frac{\rho a^{\rho} b^{\rho}\left(b^{\rho}-a^{\rho}\right)}{2} \Lambda_{12}^{1 / k}\left(\Lambda_{13}\left|f^{\prime}\left(b^{\rho}\right)\right|^{l}+\Lambda_{14}\left|f^{\prime}\left(a^{\rho}\right)\right|^{l}\right)^{1 / l}
$$

where

$$
\begin{aligned}
\Lambda_{12}= & \frac{1}{\rho 2^{(k \rho-k+1) / \rho}} B\left(\frac{k \rho-k+1}{\rho}, \alpha k+1\right) \\
& +\frac{1}{\rho 2^{\rho}}{ }_{2} F_{1}\left(\frac{k+\rho-k \rho-1}{\rho}, 1 ; \alpha k+2 ; \frac{1}{2}\right) \\
\Lambda_{13}= & \frac{1}{(\rho+1) b^{2 \rho l}}{ }_{2} F_{1}\left(2 l, \frac{\rho+1}{\rho} ; \frac{2 \rho+1}{\rho} ; 1-\frac{a^{\rho}}{b^{\rho}}\right) \\
\Lambda_{14}= & \frac{\rho}{(\rho+1) b^{2 \rho l}}{ }_{2} F_{1}\left(2 l, \frac{1}{\rho} ; \frac{2 \rho+1}{\rho} ; 1-\frac{a^{\rho}}{b^{\rho}}\right) .
\end{aligned}
$$

Proof. Let $A_{t}=t^{\rho} a^{\rho}+\left(1-t^{\rho}\right) b^{\rho}$. From Lemma 1, Lemma 2, Hölder inequality and the harmonically convexity of $|f|^{q}$, we have

$$
\begin{aligned}
\left|I_{f}(g ; \alpha, a, b)\right| \leq & \frac{\rho a^{\rho} b^{\rho}\left(b^{\rho}-a^{\rho}\right)}{2} \int_{0}^{1} \frac{\left|t^{\rho \alpha}-\left(1-t^{\rho}\right)^{\alpha}\right|\left|t^{\rho-1}\right|}{A_{t}^{2}}\left|f^{\prime}\left(\frac{a^{\rho} b^{\rho}}{A_{t}}\right)\right| d t \\
\leq & \frac{\rho a^{\rho} b^{\rho}\left(b^{\rho}-a^{\rho}\right)}{2}\left(\int_{0}^{1}\left|t^{\rho \alpha}-\left(1-t^{\rho}\right)^{\alpha}\right|^{k}\left|t^{\rho-1}\right|^{k} d t\right)^{1 / k} \\
& \times\left(\int_{0}^{1} \frac{1}{A_{t}^{2 l}}\left|f^{\prime}\left(\frac{a^{\rho} b^{\rho}}{A_{t}}\right)\right|^{l} d t\right)^{1 / l} \\
\leq & \frac{\rho a^{\rho} b^{\rho}\left(b^{\rho}-a^{\rho}\right)}{2}\left(\int_{0}^{1}\left|\left(2 t^{\rho}-1\right)\right|^{\alpha k} t^{k(\rho-1)} d t\right)^{1 / k} \\
& \times\left(\int_{0}^{1} \frac{1}{A_{t}^{2 l}}\left[t^{\rho}\left|f^{\prime}\left(b^{\rho}\right)\right|^{l}+\left(1-t^{\rho}\right)\left|f^{\prime}\left(a^{\rho}\right)\right|^{l}\right] d t\right)^{1 / l} \\
\leq & \frac{\rho a^{\rho} b^{\rho}\left(b^{\rho}-a^{\rho}\right)}{2} K_{6}^{1 / k}\left(K_{7}\left|f^{\prime}\left(b^{\rho}\right)\right|^{l}+K_{8}\left|f^{\prime}\left(a^{\rho}\right)\right|^{l}\right)^{1 / l} .
\end{aligned}
$$

where

$$
\Lambda_{12}=\int_{0}^{1}\left|\left(2 t^{\rho}-1\right)\right|^{\alpha k} t^{k(\rho-1)} d t
$$




$$
\begin{aligned}
= & \int_{0}^{1 / 2^{1 / \rho}}\left(1-2 t^{\rho}\right)^{\alpha k} t^{k(\rho-1)} d t+\int_{1 / 2^{1 / \rho}}^{1}\left(2 t^{\rho}-1\right)^{\alpha k} t^{k(\rho-1)} d t \\
= & \frac{1}{\rho 2^{(k \rho-k+1) / \rho}} B\left(\frac{k \rho-k+1}{\rho}, \alpha k+1\right) \\
& +\frac{1}{\rho 2^{\rho}} 2 F_{1}\left(\frac{k+\rho-k \rho-1}{\rho}, 1 ; \alpha k+2 ; \frac{1}{2}\right), \\
\Lambda_{13}= & \int_{0}^{1} t^{\rho} A_{t}^{-2 l} d t \\
= & \frac{1}{(\rho+1) b^{2 \rho l}}{ }_{2} F_{1}\left(2 l, \frac{\rho+1}{\rho} ; \frac{2 \rho+1}{\rho} ; 1-\frac{a^{\rho}}{b^{\rho}}\right) \\
\Lambda_{14}= & \int_{0}^{1}\left(1-t^{\rho}\right) A_{t}^{-2 l} d t \\
= & \frac{\rho}{(\rho+1) b^{2 \rho l}}{ }_{2} F_{1}\left(2 l, \frac{1}{\rho} ; \frac{2 \rho+1}{\rho} ; 1-\frac{a^{\rho}}{b^{\rho}}\right)
\end{aligned}
$$

So, if we use (3.30)-(3.32) in (3.28), we obtain desired result.

Remark 7. In Theorem 10, taking limit $\rho \rightarrow 1$ we obtain Theorem 9 in [5].

\section{REFERENCES}

[1] S. Belarbi and Z. Dahmani, "On some new fractional integral inequalities," J. Inequal. Pure Appl. Math., vol. 10, no. 3, p. 5, 2009.

[2] Z. Dahmani, "New inequalities in fractional integrals," IJNS, International Journal of Nonlinear Sciences, vol. 9, no. 4, pp. 493-497, 2010.

[3] Z. Dahmani, L. Tabharit, and S. Taf, "New generalisations of gruss inequality using riemannliouville fractional integrals," Bull. Math. Anal. Appl., vol. 2, no. 3, pp. 93-99, 2010.

[4] I. Iscan, "Hermite-hadamard type inequalities for harmonically convex functions," Hacet. J. Math. Stat., 2014.

[5] I. Iscan and S. Wu, "Hermite-hadamard type inequalities for harmonically convex functions via fractional integrals," Applied Mathematics and Computation, vol. 238, pp. 237-244, 2014, doi: 10.1016/j.amc.2014.04.020.

[6] U. Katugampola, "New approach to generalized fractional derivatives," Bull. Math. Anal. Appl., vol. 6, no. 4, pp. 1-15, 2014.

[7] A. Kilbas, H. Srivastava, and J. Trujillo, Theory and applications of fractional differential equations. Amsterdam: Elsevier, 2006.

[8] M. A. Noor, M. U. Awan, and K. I. Noor, "On some inequalities for relative semi-convex functions," Journal of Inequalities and Applications, vol. 332, 2013.

[9] M. A. Noor, K. I. Noor, and M. U. Awan, "Generalized convexity and integral inequalities," Applied Mathematics and Information Sciences, vol. 9, no. 1, pp. 233-243, 2015, doi: 10.12785/amis/090129.

[10] A. Prudnikov, Y. Brychkov, and O. Marichev, Integral and series In: Elementary Functions. vol. 1. Nauka, Moscow, 1981.

[11] E. Rainville, Special Functions. The Mcmillan Company, New York, 1960. 
[12] M. Z. Sarikaya, E. Set, H. Yaldiz, and N. Basak, "Hermite-hadamard's inequalities for fractional integrals and related fractional inequalities," Math. Comput. Model., vol. 57, pp. 2403-2407, 2013, doi: 10.1016/j.mcm.2011.12.048.

[13] E. Set, A. Akdemir, and B. Çelik, "On generalization of fej'er type inequalities via fractional integral operator,” Filomat, vol. 32, no. 16, pp. 5537-5547, 2018, doi: 10.2298/FIL1816537S.

[14] E. Set, A. Akdemir, and I. Mumcu, "Ostrowski type inequalities involving special functions via conformable fractional integrals," J. Adv. Math. Stud., vol. 10, no. 3, pp. 386-395, 2017.

[15] E. Set, A. Akdemir, and I. Mumcu, "Hadamard's inequality and its extensions for conformable fractional integrals of any order $\alpha>0$," Creat. Math. Inform., vol. 27, no. 2, pp. 197-206, 2018.

[16] E. Set and B. Çelik, "Fractional hermite-hadamard type inequalities for quasi-convex functions," Ordu Univ. J. Sci. Tech., vol. 6, no. 1, pp. 137-149, 2016.

[17] E. Set, I. Iscan, and F. Zehir, "On some new inequalities of hermite-hadamard type involving harmonically convex functions via fractional integrals," Konuralp J. Math., vol. 3, no. 1, pp. 4255,2015

[18] E. Set, M. Noor, M. Awan, and A. Gözpınar, "Generalized hermite-hadamard type inequalities involving fractional integral operators," Journal of Inequalities and Applications, vol. 2107, no. 169, pp. 1-10, 2017, doi: 10.1186/s13660-017-1444-6.

[19] E. Set, M. Z. Sarikaya, M. E. Ozdemir, and H. Yildirim, "The hermite-hadamard's inequality for some convex functions via fractional integrals and related results," JAMSI, vol. 10, no. 2, pp. 69-83, 2014, doi: 10.2478/jamsi-2014-0014.

Authors' addresses

\section{İlker Mumcu}

Ordu University, Department of Mathematics, Faculty of Science and Arts, Ordu, Turkey

E-mail address: mumcuilker@msn.com

Erhan Set

Ordu University, Department of Mathematics, Faculty of Science and Arts, Ordu, Turkey

E-mail address: erhanset@yahoo.com

\section{Ahmet Ocak Akdemir}

Ağr1 İbrahim Çeçen, Department of Mathematics, Faculty of Science and Arts, Ağrı, Turkey

E-mail address: ahmetakdemireagri.edu.tr 